\title{
An Automatic Colour Sensor based Urine Analysis Device to Detect CKD for Mass Screening Application
}

\author{
Nikhil Thomas ${ }^{1}$, Sachin Katti ${ }^{2}$, Sumir R M ${ }^{3}$, Lakshmi Priyanka ${ }^{4}$, Pratiksha Umesh ${ }^{5}$ \\ ${ }^{2}$ Assistant Professor, ${ }^{1,3,4,5}$ UG Students, \\ Medical Electronics Department, MVJ College of Engineering \\ nikhilthomas207@gmail.com
}

\begin{abstract}
Chronic kidney disease (CKD) is a condition characterized by a loss of kidney function gradually over a period of time with five stages. Usually, in the initial stages, the disease appears to be asymptomatic, as a result diagnosis of the disease in the early stages is unlikely. When diagnosed in the early stages (stages 1-2) the disease is reversible. For early diagnosis of the disease, we use an automatic system consisting of sensor TCS3200 and a urine strip. Bluetooth module is used to transfer the data to the mobile or computer for further analysis. The sensor detects the change in color on the strip when the sample is introduced. Change in color of the strip is due to the presence of albumin in urine, which is the primary biomarker considered to detect the early stage of kidney dysfunction.
\end{abstract}

\section{INTRODUCTION}

Chronic Kidney Disease (CKD) is a serious clinical condition characterized by loss of kidney function over the course of time. Persistence of impaired kidney function over a period of 3 months can have major health implications [1]. Stages 1-3 of CKD are usually asymptomatic (GFR \&gt; 30 $\mathrm{mL} / \mathrm{min} / 1.73 \mathrm{~m}^{2}$ ). By stage 4 or 5 , some of the clinical implications manifest as anemia, peripheral neuropathy, malnutrition, dry skin, nausea, and diarrhea and platelet dysfunction. Hypertension, Cystic kidney disease, Diabetic Kidney Disease, Renal Vein Thrombosis can all be viewed as causative factors of Chronic Kidney Disease. Owing to the high prevalence, mortality and morbidity rates, CKD is often observed as a major public health issue. About $17 \%$ of the people living in India are affected by CKD, which approximately amounts to 224 million Indians. This essentially indicates that 1 in 6 people are affected by CKD. Despite this alarming statistic, CKD is utterly disregarded as an area of concern when compared to other communicable diseases such as infant and maternal mortality [2]. End-stage renal disease, cardiovascular risk and high mortality rate are attributed as serious clinical implications of Chronic Kidney Disease. Laboratory tests, imaging studies, physical examinations are used for screening of CKD and other diseases. A screening procedure is generally followed by detailed testing, specific treatment, and a follow-up procedure fixated on turning around, slowing down or averting the progression of the disease [3]. Operational difficulty, social stigma, resource constraints and cost of treatment are some of the issues related to screening.
Difficulty in locating the physician's office, scarcity of computer-based diagnostic systems, economic considerations, lack of understanding with regard to the clinical condition and inadequate information about clinical guidelines are some of the barriers for screening of CKD [3]. In the case of CKD, Micro albuminuria is not filtered out by the process of ultrafiltration. Due to lack of a reliable diagnostic procedure, this goes undetected in the asymptomatic stages. This is indicative of the impairment in kidney function. The objective of this paper is to implement an easy, cost-effective method to screen a population with asymptomatic chronic kidney disease and initiate therapy to reduce the risk of cardiovascular events and end-stage renal disease in an outreach camp setting. To achieve this we will be using Urine test strips, the color sensor (TCS3200) interfaced with a microcontroller and an Arduino board. The proposed system is able to detect the change in color in the urine strips, which is indicative of albuminuria content in urine. The sensor values are transferred to the mobile application using a Bluetooth module.

\section{OVERVIEW OF CHRONIC KIDNEY DISEASE}

This section of the paper is intended to discuss the pathophysiology, risk factors, stages of CKD, negative outcomes and importance of screening. The exploration of present technologies and future developments is a part of this discourse. The analysis is extended so as to explain why the proposed system is better by comparing it with existing screening methods.

\section{A.Pathophysiology :}

There are 1 million nephrons in the kidney. Even in the case renal injuries, the kidney can take care of GFR. In spite of increasing destruction of nephrons, the healthy nephrons show hyper filtration and hypertrophy. This Characteristic ability of nephron allows for the normal flow of plasma solutes, only after the decrease in GFR by $50 \%$ there is a measurable increase in the urea and creatinine levels in the Plasma [4].. Kidney dysfunction is indicated by an increase in plasma creatinine value in a patient $(0.6 \mathrm{mg} / \mathrm{dL}$ to 1.2 $\mathrm{mg} / \mathrm{dL}$ ), even though this value is still within the normal adult range. The capillaries may be damaged due to the increased glomerular capillary pressure. 


\section{Stages of CKD:}

The progression of Kidney Dysfunction can be broadly classified into five stages, from an asymptomatic mild damage to end-stage kidney failure. The stages of kidney disease can be categorized based on their ability to filter out waste which in CKD decreases as the disease progresses. In the early stages of kidney disease, your kidneys are still able to filter out waste from the blood. In the final stages, the kidneys have to work harder to filter out the waste.

Stage 1: There is moderate Kidney harm in stage 1 Chronic Kidney Disease and there are no signs and symptoms. Physician consultation is vital in preventing the further loss of kidney malfunction. The kidneys are healthy if the GFR is more than 90 . Even though the GFR is normal there might be signs of CKD

Stage 2: There is a slight kidney damage in stage 2 kidney disease. If the GFR is between 60 and 89 then the kidney is in good condition. At this stage is advised to take care of the disease and prevent it from getting worst.

Stage 3: The initial loss of kidney functionality is indicated by stage 3 CKD. There is a variation in the GFR based on the subcategories in stage 3 . If it is between 45 to 59 then it is stage $3 \mathrm{a}$ and if between 30 and 44 it is stage $3 \mathrm{~b}$. Bone disease, anemia are the further clinical complications.

Stage 4: The kidneys are seriously damaged in stage 4 . This is a critical stage and should be considered more seriously [9]. At this stage, the kidney has lost its ability tofunction properly. Waste building up in the body is the other complication at stage 4 .

Stage 5 of CKD indicates Kidney failure and there are severe symptoms. The normal kidney function is disturbed by the accumulation of waste and toxins in the blood. The patient has to undergo a kidney transplantation or dialysis once the kidneys have completely failed.

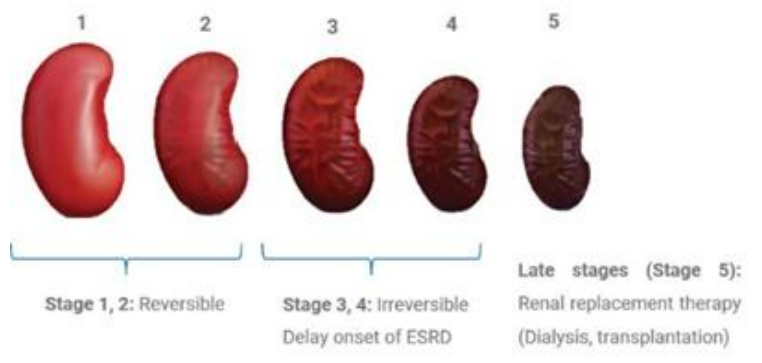

Fig 1: Stages of Kidney Diseases

\section{BIOMARKERS}

\section{A. Proteinuria and Albuminuria:}

The earliest Biomarkers of kidney Dysfunction in patients suffering from diabetes, glomerular disease and hypertension are Albuminuria and Proteinuria. Increased excretion of serum proteins and Albumin is Proteinuria. Urinary tract infection, hemodynamic stress, hyperglycemia can cause a transient increase in excretion of albumin and Total Protein. The persistent levels of Proteinuria over a period of three months indicate kidney damage. The important determinant of progression of End Stage Renal Disease (ESRD) is Proteinuria [13]. The screening program is initiated for the individuals who have proteinuria secretion in kidney .a study was initiated by Japanese to understand the relationship between increased ESRD levels and urinalysis results. Micro albuminuria (MA) is recognized as a crucial risk issue for cardiovascular and renal complications in diabetes. Micro albuminuria was ab-initio found to predict consequent overt proteinuria (dipstick positive, or $>300 \mathrm{mg} / 24 \mathrm{~h}$ ), that successively predicted loss of GFR (1-3) [14]. From the strength of those relationships, it's often been assumed that micro albuminuria and overt proteinuria are requisite first and second steps on one pathway that results in loss of GFR and ESRD. A meta-analysis of eight cohorts of 845,125 general and risky individuals confirms increased risk for ESRD in those with stage 3 chronic kidney disease that is GFR about $60 \mathrm{ml} / \mathrm{min}$ and in individuals with symptom the least bit levels independent of traditional cardiovascular risk issue .Because medical treatment reducing the chance of complications is accessible, urine albumin (UA) is very suggested as a screening test for diabetes patients, though it's progressively recommended also for the early diagnosis of nephritic unwellness and cardiovascular risk stratification[15]. The tests for screening albumin are less expensive and have high sensitivity and specificity for chronic kidney disease caused by Diabetes. The occurrence of cardiovascular disease is indicative of higher levels of micro albuminuria. The study conducted by the Japanese researchers showed that dipstick Proteinuria test may be used to detect the micro albuminuria and gave positive results.

\section{B. Creatinine}

Serum creatinine is a significant marker of renal well-being .Creatinine itself is produced through an organic framework including creatinine, phosphocreatine, and adenosine triphosphate estimating serum creatinine is a straightforward test, and it is the most regularly utilized pointer of renal capacity. Creatinine isn't constantly illustrative of a genuine 


\section{PROPOSED SYSTEM}

The current issues faced in mass screening have operational difficulty outside the laboratory setting, cultural stigma, resource constraints and are cost ineffective. These issues can be compensated using a portable urine analyzer specifically designed for mass screening application that incorporates TCS3200(color sensor), Urine test strips, Arduino board and an Arduino software to interface the board with the sensor. The detailed description of urine strips, the color sensor is further explained.

\section{A. Urine Strips}

A Urine strip is a basic diagnostic tool used to determine pathological variations in the patient's urine sample. The test results are usually obtained within a timeframe ranging from a few seconds to minutes. There can also be instances where it could exceed the mentioned limit. The various proteins present are Aldohexose, Hemoprotein, Ketones, Hematoidin, Stercobilinogen. Parameters like $\mathrm{pH}$ and relative density are also considered during urinalysis. Since our focus is on the early detection of kidney failure, one of the best biomarkers that can be utilized is the protein albumin (Albuminuria) in urine. Proteinuria is related to the presence of abnormal quantities of protein in the urine, which leads to the formation of urinary macromolecules. Urinary organ impairment can be viewed as a direct consequence of this phenomenon. In general, the color of the test strip changes to green when it comes in contact with the sample. This is indicative of albumin content in urine. Based on this observation, a color sensor is used to detect the color change on the strip, which in turn indicates the presence of protein in the urine sample.

The flowchart below portrays the operational flow of the whole system which includes sample collection, color detection of the strip which results in the early detection of renal disease.

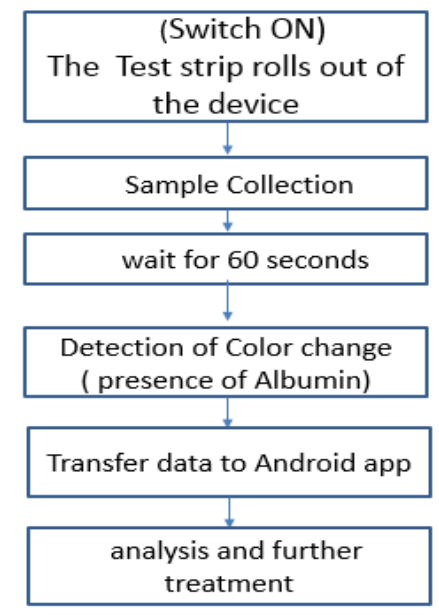

Fig.5. Operational flow of proposed method

Vol. 2 (12), June 2019, www.ijirase.com

\section{B. TCS 3200 Colour Sensor}

The sensor TCS3200 senses light using an 8x8 array of photodiodes, which converts light energy to current. The current is then converted into square wave frequencies using a current to frequency converter whose frequency is directly proportional to light intensity. Finally, by exploiting the Arduino Board, a square wave is observed as a result. This exemplifies color detection by means of a sensor.

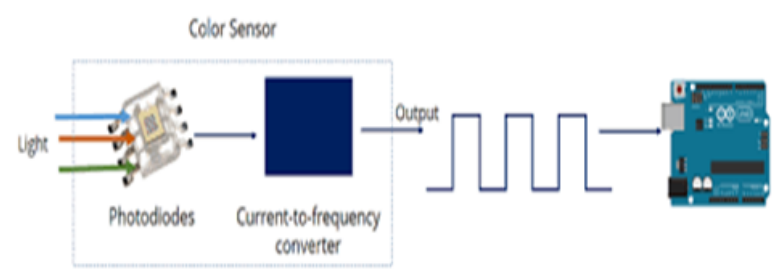

Fig.1: Current to Frequency Conversion

The photodiodes have 3 different color filters - Red, green and blue, each of which are sixteen in number. The other sixteen photodiodes are clear with no filters. Each of the sixteen photodiodes is arranged in a parallel manner. The required photodiode type can be derived by using the two control pins S2 and S3. The sensing element has 2 additional management pins, S0 and S1 that are used for scaling of the output frequency. The frequency can be scaled to three different preset values of $2 \%, 20 \%, 100 \%$. This frequencyscaling factor allows the output of the sensor to be optimized for numerous embedded systems. Circuit connection made between color sensor TCS3200 and Arduino board is shown below.

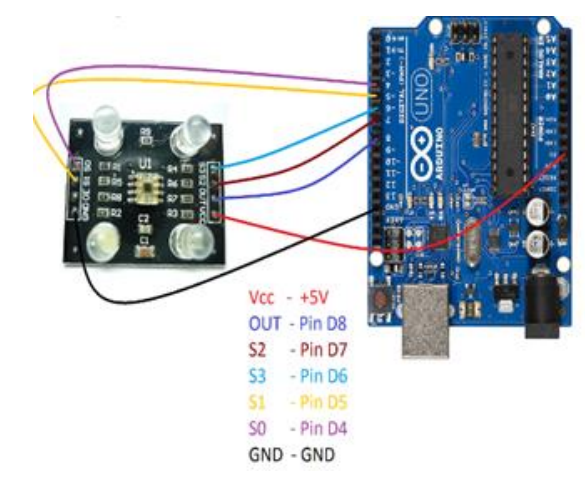

Fig.6: Color Sensor interfaced with Arduino

\section{RESULTS}

The device consists of an automatic test strip roll system. When the device is turned on the strip rolls out. After the urine sample is collected the strip rolls back. The color sensor placed inside the device detects the change in color which indicates the presence of albumin in the urine. A multiparameter urine test strip is used to measure various parameters . Egg white is used to detect the presence of protein for the experimentation purpose. The TCS 3200 color 
sensor was able to detect the change in color from yellow to green. The results are shown in the table below. An android application is developed for the data transfer and analysis.

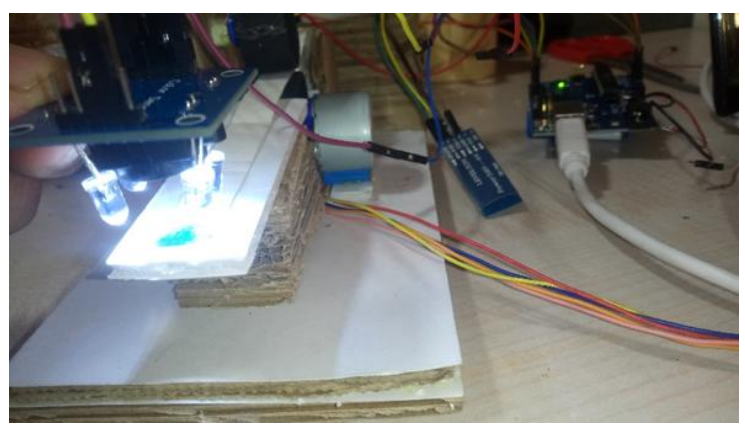

Fig 7. Proposed Concept of the System

\begin{tabular}{l|l|}
\hline HC-O5 & (i) \\
\hline protein: & green color \\
\hline Sensor 2: & 4444.0 \\
\hline & \\
\hline Sensor 3: & $1234 \mathrm{hPa}$ \\
\hline
\end{tabular}

Fig 8. Android Application

TABLE I. COLOR DETECTION USING SENSOR

\begin{tabular}{|l|l|l|}
\hline \multicolumn{1}{|c|}{ PARAMETRS } & \multicolumn{1}{|c|}{$\begin{array}{c}\text { COLOUR } \\
\text { DETECTED }\end{array}$} & \multicolumn{1}{c|}{ RESULT } \\
\hline EGG WHITE & GREEN & ALBUMIN PRESENT \\
\hline WATER & YELOW & $\begin{array}{l}\text { ALBUMIN } \\
\text { ABSENT }\end{array}$ \\
\hline
\end{tabular}

\section{CONCLUSION AND FUTURE SCOPE}

Chronic kidney disease is a gradual loss of kidney function over 5 stages. The disease is completely reversible when detected in the initial two stages. We use a portable and reliable system consisting of light sensor TCS3200 and a urine strip to detect CKD in the initial stages. The biomarker considered is albumin detected through the change in color in the urine strip. With the detection of CKD in the initial stages, further deterioration of the kidney is prevented also risks of diabetes, hypertension and cardiovascular disease is reduced

In the future, we aim to modify the system for measuring other parameters such as $\mathrm{pH}$, specific gravity, glucose etc. This system can be implemented to be a homebased system. A dedicated Android application developer can keep a track of various parameters of kidney obtained from patient data. An IOT system can be enforced to trace the varied different vitals of the kidney that facilitate in early detection of CKD.

\section{REFERENCES}

[1] KDIGO 2012 Clinical Practice Guideline for the Evaluation and Management of Chronic Kidney Disease. January 2013

[2] Abraham G, Varghese S, Thandavan T, Iyengar A, Fernando Naqvi SA, Sheriff R, Ur-Rashid H, Gopalakrishnan N, Kafle RK," Chronic kidney disease hotspots in developing countries inSouth Asia". Clin Kidney J 9: 135-141, 2016

[3] Berns, Jeffrey S. "Routine screening for CKD should be done in asymptomatic adults... selectively." Clinical Journal of the American Society of Nephrology 9.11 (2014): 1988-1992.

[4] NIDDK Causes of CKD https://www.niddk.nih.gov/healthinformation/kidney-disease/chronic-kidney-disease-ckd/causes Accessed 2/7/2018

[5] American Diabetes Association (ADA). 2010. Standards pf medical care in diabetes-2010. Diabetes Care. 33(1): 11-61.

[6] Hjelmesæth J, Trond J, Egeland T, Hagen M, Hartmann A: Response to Montori (Letter). Diabetes Care 25:1667

[7] Vanholder R1, Massy Z, Argiles A, Spasovski G, Verbeke F, Lameire N" Chronic kidney disease as cause of cardiovascular morbidity and mortality" DOI: 10.1093/ndt/gfh813I.

[8] http://www.kidneyfund.org/kidney-disease/chronic-kidneydisease-ckd/stages-of-chronic-kidney-disease

[9] https://www.freseniuskidneycare.com/about-chronic-kidneydisease/stages/chronic-kidney-disease-ckd

[10] Modi, Gopesh et al." Incidence of ESRD in India Kidney International, Volume 79, Issue 5 , 573 DOI: https://doi.org/1 0.1038/ki.2010.477Y.

[11] http://www.kidneyfund.org/kidney-disease/chronic-kidneydisease-ckd/complications/heart-disease.

[12] Couser, William G., et al. "The contribution of chronic kidney disease to the global burden of major noncommunicable diseases." Kidney international 80.12 (2011): 1258-1270.

[13] Iseki K, Ikemiya $Y$, Iseki C et al. Proteinuria and the risk of developing end-stage renal disease. Kidney International. 2003; 63: 1468-74

[14] de Boer, Ian H., and Michael W. Steffes. "Glomerular filtration rate and albuminuria: twin manifestations of nephropathy in diabetes." Journal of the American Society of Nephrology 18.4 (2007): 1036-1037.

[15] Brantsma, Auke H., et al. "Cardiovascular and renal outcome in subjects with K/DOQI stage 1-3 chronic kidney disease: the importance of urinary albumin excretion." Nephrology Dialysis Transplantation 23.12 (2008): 3851-3858. 